\title{
Next-generation space telescope sets course for 2010 launch
}

Tony Reichhardt, Washington

It has a basic design, a team of scientists to oversee it and a new name. Now one of astronomy's most challenging projects is poised to begin development.

NASA announced last week that the Next Generation Space Telescope will be named after James Webb, the administrator who led the agency during its glory days of the 1960s, which culminated in the Moon landing of July 1969. The James Webb Space Telescope, which is designed to continue operating for at least five years after its launch in 2010, is expected to cost $\$ 2.8$ billion.

The telescope, which will have a diameter of 6 metres and be optimized to view infrared light, topped the US astronomy community's ten-year wish-list of projects released in 2000 (see Nature 405, 381; 2000). A large light-collecting area and sensitive detectors will enable it to see galaxies that are fainter,

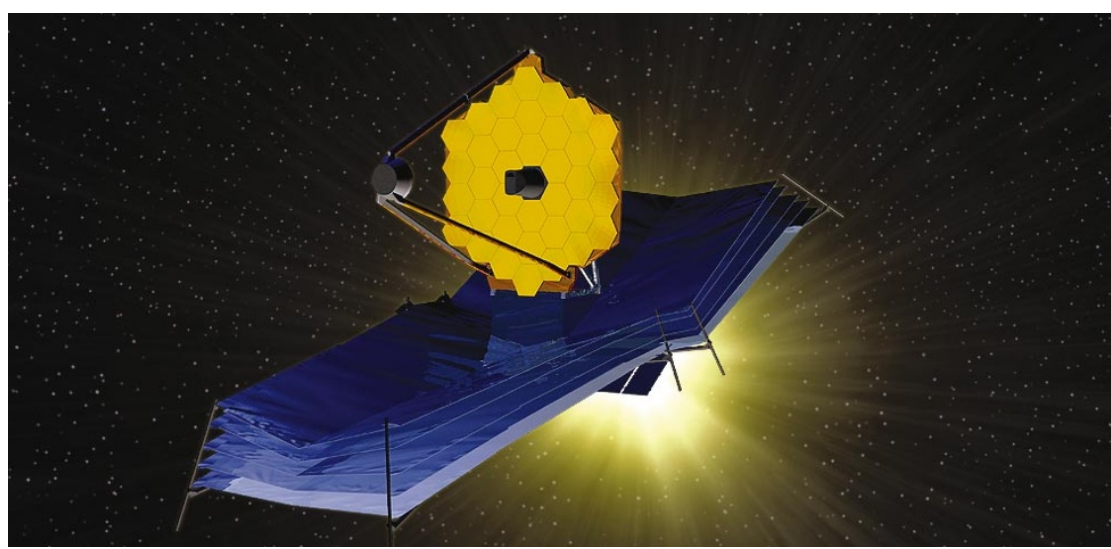

Petal power: an artist's impression of the new telescope, whose mirror will open like a flower.

older and farther away than any seen by the Hubble Space Telescope. Astronomers are hoping they will be able to learn how large

\section{US return may boost 'S' in UNESCO}

\section{Jonathan Knight}

The United States has promised to rejoin the United Nations Educational, Scientific and Cultural Organization (UNESCO), nearly two decades after it severed ties with the organization.

President George W. Bush made the announcement during a speech to the UN General Assembly in New York on 12 September, saying that the organization had been sufficiently reformed. The United States withdrew its funding and staff in 1984 on the grounds that UNESCO had become too bureaucratic and politicized.

UNESCO, which was founded after the Second World War to promote international collaboration on scientific and cultural issues, has a relatively small science budget of about US\$50 million. It runs several influential international science bodies, such as the Intergovernmental Oceanographic Commission, which helps to coordinate ocean research.

The decision was welcomed by US scientists who had urged their country to rejoin the organization.

"It seemed rather anomalous that a great country like this wouldn't support it," says James Cronin, a physicist at the University of Chicago and a member of the Pierre Auger Observatory, a cosmic-ray detector currently under construction in Argentina (see Nature 419, 12-14; 2002) that UNESCO has helped to fund. Cronin was also one of 37 Nobel laureates who unsuccessfully petitioned President Bill Clinton to rejoin the organization in 1993.

The impact of the decision remains unclear, however. UNESCO's budget will not immediately increase, as the United States' contribution of $\$ 60$ million a year will be subtracted from the total payments made by other countries. But the move could allow US researchers to push for the organization to divert more of its resources to scientific projects.

"The ' $S$ ' stands for science, but it's the smallest 'S' you can imagine," says Cronin. structures formed in the early Universe, and to observe the birth of stars and planets with unprecedented clarity.

This won't be easy. The design incorporates several untested ideas, including the first placement of a large observatory at the L2 point, 1.5 million kilometres away from Earth in the opposite direction to the Sun. As a result of the combined influence of the gravitational attraction of the Sun and Earth, objects stationed at L2 orbit the Sun once a year and remain in roughly the same position relative to Earth.

L2 is colder than Hubble's orbit around Earth, so the Webb can cool itself using a sunshield instead of bulky and expensive mechanical systems. Stationing the telescope at L2 also makes it easier to target than Hubble, which has to avoid pointing at the Earth or Moon. This should bring the operating costs of the new telescope down to around a quarter of those required for Hubble, says project manager Bernard Seery of NASA's Goddard Space Flight Center.

L2 does have one main disadvantage: a telescope stationed there cannot be repaired or upgraded by astronauts, as Hubble can. If a crucial onboard system fails and cannot be fixed by radio command, the mission is likely to end.

The winning design for the telescope which was put forward by an industrial consortium led by TRW Space and Electronics 
of Redondo Beach, California and Ball Aerospace of Boulder, Colorado borrows from research on large space mirrors developed for military satellites. The segmented primary mirror divides into three panels, which, once in space, will unfold like flower petals. The technical difficulty of testing this system is the main reason for a slip in the launch date from 2008 to 2010.

Once in space, the mirror, which will be made of glass or beryllium, will be tested and adjusted every few weeks to compensate for any changes in shape caused, for example, by heating.

These and other new technologies have pushed up costs, however. Last year, astronomers reluctantly agreed to NASA's proposal to decrease the size of the mirror from 8 to 6 metres, reducing the telescope's resolving power by $25 \%$ and its light-collecting ability by $44 \%$. An orbital test of a scaled-down mirror was also scrapped.

Some outside observers worry that the current budget is still too small, but Seery says his team is far more savvy about the technological challenges and true costs than it was.

Others say that it is right to push the state of the art forward with the planned new telescope.

"These kinds of telescopes only come along once every decade or so," says Jonathan Lunine, a planetary scientist at the University of Arizona in Tucson and a member of the Webb's recently appointed Science Working Group, which will meet for the first time next week. "Because of the size and the scope of this mission, the opportunity to do new technology is really there."

http://ngst.gsfc.nasa.gov

\section{Prion research stepped up as fear grows of deer disease}

\section{Rex Dalton and Erika Check}

As concern mounts in the western United States about a relative of mad cow disease found among deer and elk, federal agencies are boosting research into this family of fatal neurodegenerative conditions.

Awareness of chronic wasting disease (CWD) has risen in the past few years. The disease, present in the Rocky Mountain states since the 1960s, has recently been found in the midwest (see Nature 416, 569-570; 2002). Officials are also concerned that mad cow disease might enter the United States.

CWD, mad-cow disease and the human form, Creutzfeldt-Jakob disease (CJD), are all thought to be caused by infectious prions - misshapen forms of a protein that can convert the normal version to their own rogue shape.

There is no evidence that CWD has caused human disease, but this remains a possibility as $60 \%$ of people in the affected region eat venison or elk. Fears about CWD have been fuelled by reports of venisoneaters who have died with neurological symptoms, although none was found to have had a prion disease.

The Department of Defense is leading the push for more research with its National Prion Research Program. The Institute of Medicine held a meeting in Washington on 12-13 September to advise on priorities for the first grants, worth $\$ 42.5$ million.

High on the agenda was the development of better diagnostic tests that could reveal infection before symptoms emerge. Cases of prion disease are currently confirmed

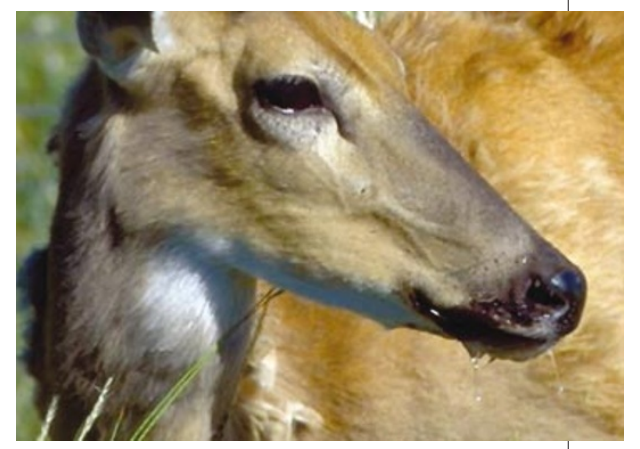

Waste concern: can disease pass to humans from animals such as this infected white-tailed deer?

by taking biopsies of tonsils or brain tissue.

The Department of Defense also wants to award grants to increase the size of the small US prion-research community. Researchers are put off by biosafety requirements and may be fearful of working with prions, says Pierluigi Gambetti of Case Western Reserve University in Cleveland, Ohio. "The reality is that you have to pay people more to work in your laboratories," he says.

Gambetti heads the National Prion Disease Pathology Surveillance Center, which examines cases of neurological disease and maintains CJD tissue samples. Its budget is to rise fourfold to $\$ 1$ million next year.

In addition, the National Institutes of Health wants to create a repository for reagents used in prion research. It is also considering creating a central repository for antibodies, which are necessary for the development of diagnostic tests.

\section{Japan celebrates safe launch after string of problems}

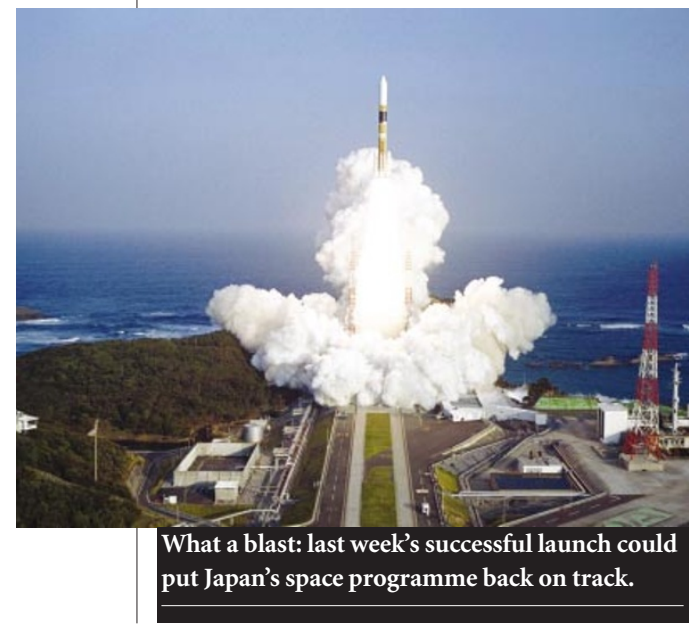

David Cyranoski, Tokyo

The successful launch of Japan's H-IIA rocket last week provided a welcome boost to the country's troubled rocket programme, before a crucial international launch later this year.

On 10 September, the National Space Development Agency of Japan (NASDA) used H-IIA to launch satellites designed to conduct data-relay and zero-gravity research. The H-IIA series and its predecessor, the $\mathrm{H}$-II, have had several failures. The most recent occurred in February, when the rocket failed to release a $¥ 600$-million (US\$4.9-million) satellite designed to measure the impact of high temperatures during re-entry.

The next H-IIA launch, scheduled for later this year, will be a major test. It will carry the Advanced Earth Observing Satellite II, with detectors made by NASDA, NASA and the CNES, France's national space agency. They will monitor chlorophyll, water vapour, sea surface temperature, sea ice and ocean wind velocity.

The mission will also include Australia's first scientific satellite launch for 30 years. FedSat, designed by Australia's Cooperative Research Centre for Satellite Systems, will carry out engineering, communication and science experiments.

NASDA hopes that more successes will help to establish Japan's space programme as commercially viable. It plans to turn over operations of the H-IIA to Mitsubishi Heavy Industries in the next two years. 